\title{
Neutrophil CD64 as a diagnostic marker for neonatal sepsis: Meta-analysis
}

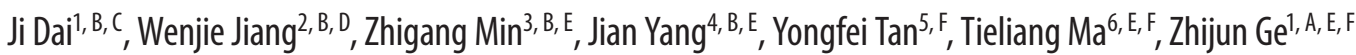 \\ ${ }^{1}$ Department of Critical Care Medicine, the Affiliated Yixing Hospital of Jiangsu University, Yixing, Jiangsu, China \\ 2 Department of Anesthesiology, the Affiliated Yixing Hospital of Jiangsu University, Yixing, Jiangsu, China \\ ${ }^{3}$ Department of Radiology, the Affiliated Yixing Hospital of Jiangsu University, Yixing, Jiangsu, China \\ ${ }^{4}$ Department of Oncology, the Affiliated Yixing Hospital of Jiangsu University, Yixing, Jiangsu, China \\ ${ }^{5}$ Department of Cardiac \& Thoracic Surgery, the Affiliated Yixing Hospital of Jiangsu University, Yixing, Jiangsu, China \\ ${ }^{6}$ Central Laboratory, the Affiliated Yixing Hospital of Jiangsu University, Yixing, Jiangsu, China \\ A - research concept and design; B - collection and/or assembly of data; $C$ - data analysis and interpretation; \\ $D$ - writing the article; $E$ - critical revision of the article; $F$ - final approval of article
}

Address for correspondence

Zhijun Ge

E-mail:staff789@yxph.com

\section{Funding sources}

This work was supported by the Fund of Science and Technology of Yixing (2013-21), the Fund of Clinical Science and Technology of Wuxi (ML201304) and the Fund of Six Best Talents of Jiangsu (WSN-024).

\section{Conflict of interest}

none declared

Received on May 19, 2015

Revised on June 3, 2015

Accepted on July 10, 2015
DOI

$10.17219 /$ acem/58782

\section{Copyright}

Copyright by Author(s)

This is an article distributed under the terms of the

Creative Commons Attribution Non-Commercial License

(http://creativecommons.org/licenses/by-nc-nd/4.0/)

\begin{abstract}
Background. Neutrophil CD64 (nCD64) is a promising marker for diagnosing bacterial infections. Several studies have investigated the performance of nCD64 for diagnosing neonatal sepsis and the results are variable. Interest in nCD64 for detecting serious bacterial infections is increasing rapidly.

Objectives. The aim of the present study was to carry out a meta-analysis to systematically evaluate the diagnostic accuracy of nCD64 in neonatal sepsis. As far as the authors know, no previous studies have undertaken this.

Material and methods. A review of studies from Pubmed, Embase and the Cochrane Library, from inception through June 2015, found 7 studies (involving 2213 neonates) fulfilling the inclusion criteria. These 7 studies were subjected to a bivariate meta-analysis of sensitivity and specificity and a summary receiveroperating characteristic (SROC) curve; $1^{2}$ was used to test heterogeneity, and the source of heterogeneity was investigated by influence analysis and meta-regression.
\end{abstract}

Results. The pooled sensitivity and specificity were $80 \%(95 \% \mathrm{Cl}, 69-88 \%)$ and $83 \%(95 \% \mathrm{Cl}, 71-90 \%)$, respectively. The area under the SROC curve (AUC) was $0.88(95 \%(1,0.85-0.91)$. The studies had substantial heterogeneity $\left(l^{2}=87.1 \%\right)$.

Conclusions. The results showed that nCD64 is a reliable biomarker for diagnosing neonatal sepsis (AUC $=0.88)$.

Key words: meta-analysis, neonatal infection, CD64, neonatal sepsis 
Neonatal sepsis is one of the most common causes of morbidity and mortality for neonates all over the world,particularly in developing countries. ${ }^{1-4}$ The incidence of neonatal sepsis is approximately 3-40 per 1000 live births, and the mortality rate ranges from $9 \%$ to $20 \% .5$

It is difficult to identify neonatal sepsis early because of a lack of specific clinical manifestations. The signs are hard to distinguish from non-infectious disorders such as maladaptation, respiratory distress syndrome and aspiration syndromes. ${ }^{7,8}$ Blood culture is regarded as the reference standard for the identification of serious bacterial infection, but it is time-consuming (2-4 days) and has high false negative/positive rates. ${ }^{9}$ This means that broad-spectrum antibiotics are applied to all suspected neonates in case of potential serious outcomes. As a result, drug-resistant strains appear and neonatal healthcare costs escalate. ${ }^{10}$

Several biochemical markers have been studied for the early diagnosis of neonatal sepsis, especially C-reactive protein (CRP) and procalcitonin (PCT). However, the specificity and the value of these markers are not sufficiently reliable. Therefore, a persistent search for better biomarkers of neonatal sepsis is still very necessary.

CD64, a high affinity receptor that binds monomeric $\mathrm{IgG}$, is normally expressed by monocytes and weakly on resting neutrophils. ${ }^{11}$ The expression of neutrophil CD64 (nCD64) is considered to be a very early phase of the host's immune response to bacterial infection, increasing about one hour after invasion. ${ }^{12,13}$ It is stimulated by inflammatory cytokines, then increases in a graded manner. nCD64 expression remains stable for more than $24 \mathrm{~h}$. The development of flow cytometric technology (FCM) has made it possible to measure nCD64 quickly and precisely with minimal blood volumes. ${ }^{14,15}$

Interest in nCD64 for detecting serious bacterial infections is increasing rapidly. The performance of nCD64 in diagnosing neonatal sepsis has been investigated in several studies and the results are variable., ${ }^{8,16,17}$ Taking all the above into consideration, The aim of the present study was to carry out a meta-analysis to systematically evaluate the accuracy of nCD64 in diagnosing neonatal sepsis.

\section{Material and methods}

\section{Search strategy and selection criteria}

Two investigators systematically searched the PubMed, Embase and the Cochrane Library databases for studies that assessed the accuracy of nCD64 in the diagnosis of neonatal sepsis.

The PubMed and the Cochrane Library combined search term used was (CD64) AND (neonatal sepsis OR neonatal infectious OR sepsis), and the Embase combined search term was (CD64) AND (sepsis). The databases were searched from their inception through June 2015.
A study was considered eligible for inclusion in the present review if it provided data on $\mathrm{nCD} 64$ for neonates with or without sepsis. Moreover, nCD64 measurement had to be performed when suspected sepsis presented before antimicrobial therapy. In the septic group, patients had either culture-proven or clinically diagnosed sepsis; in the non-septic group, neonates had benign clinical disorders. Only studies written in English were included.

Furthermore, the studies had to provide sufficient information to construct a $2 \times 2$ contingency table with false and true positives and negatives provided. All studies that involved healthy neonates and patients older than 28 days were excluded. Animal experiments, reviews, correspondences, case reports, expert opinions and editorials were excluded.

Neonatal sepsis diagnosed in the first $72 \mathrm{~h}$ of life was considered early onset sepsis (EOS); after $72 \mathrm{~h}$ it was considered late onset sepsis (LOS). The Quality Assessment of Diagnostic Accuracy Studies (QUADAS) tool was used to assess the methodological quality of the studies included. ${ }^{18}$ If agreement could not be reached, differences were resolved by a $3^{\text {rd }}$ investigator (ZM).

\section{Data extraction}

Two investigators independently extracted the data. If major discrepancies were observed between the data reported in the studies and the data calculated, the corresponding authors were contacted via e-mail with a request for the raw data. If no response was received after sending a reminder, the study was excluded.

The data extracted from the selected studies included the first author; the year of publication; the study design; the number of septic/non-septic patients; the standard of sepsis diagnosis (culture-proven, clinical); the method of nCD64 analysis; the analysis cut-off values; the number of true positive (TP), false positive (FP), false negative (FN) and true negative (TN) specimens; sensitivity/specificity and the positive/negative predictive value (PPV/NPV).

\section{Data analysis}

All the studies that evaluated nCD64 in neonates with culture-proven or clinically diagnosed sepsis in comparison with ill neonates that had other conditions were included in the analysis.

A bivariate mixed-effects regression model was performed to synthesize the pooled sensitivity, specificity, positive/negative likelihood ratios (P/N LRs) and the diagnostic odds ratio (DOR). This model did not transform pairs of sensitivity and specificity of individual studies into a single indicator of diagnostic accuracy, but ensured the two-dimensional nature of the data, taking into account any correlations between pairs of studies. A summary receiver-operating characteristic (SROC) curve was also constructed, plotting sensitivity vs specificity, and 
the area under the curve (AUC) was calculated. Statistical heterogeneity among the studies was evaluated by $\mathrm{I}^{2}$ statistics. Values of 25, 50 and $75 \%$ for the $\mathrm{I}^{2}$ test were considered low, moderate and high statistical heterogeneity, respectively. The publication bias of the included studies was assessed by Deek's funnel plot asymmetry test. The Spearman correlation coefficient between the logits of sensitivity and specificity was used to evaluate the presence of a threshold effect in the accuracy of nCD64. Fagan's nomogram was used to calculate post-test probability (PTP). All the above analyses were performed using the Midas Module in Stata software, v. 12 (Stata Corporation, College Station, USA) and Metadisc 1.4 (XI Cochrane Colloquium, Barcelona, Spain). A p-value $<0.05$ was considered statistically significant.

\section{Results}

\section{Study selection process}

The database search retrieved 308 studies. After reviewing the titles and abstracts, 266 articles were excluded, consisting of 98 duplicates, 15 case reports, 93 commentaries, 9 meta-analyses, 13 reviews, 12 meeting abstracts and poster presentations and 26 that did not investigate the diagnostic accuracy of neutrophil CD64 as a marker for sepsis. A further 35 were excluded after a full text review, leaving 7 studies for inclusion. ${ }^{19-25}$ The 35 articles included 22 in which the reference group or control group did not correspond to the definitions of the present metaanalysis, 2 that involved adult/pediatric or mixed populations and 13 for which $2 \times 2$ contingency tables could not be made (Fig. 1).

\section{Characteristics of the studies included}

Seven studies were included in the review. The 2213 neonates in these studies came from different parts of the world. Among these 2213 patients, 869 (39\%) had sepsis (culture-proven or clinical) and 1344 were non-septic but with other critical conditions. The study population sizes ranged from 32 to 1156 . All the studies were carried out in newborn intensive care units (NICUs) and nCD64 expression was measured using flow cytometry analysis. The types of study design were either prospective casecontrol or cohort studies. The quality of the 7 studies was generally high, satisfying the majority of the QUADAS criteria (Table 1).

\section{Diagnostic accuracy of nCD64}

Significant heterogeneity between studies was demonstrated $\left(\mathrm{I}^{2}=87.1 \%\right)$ for DOR. The pooled sensitivity of nCD64 for the diagnosis of neonatal sepsis was $80 \%$ (95\%CI, 69-88\%), and the specificity was $83 \%$ (95\%CI,

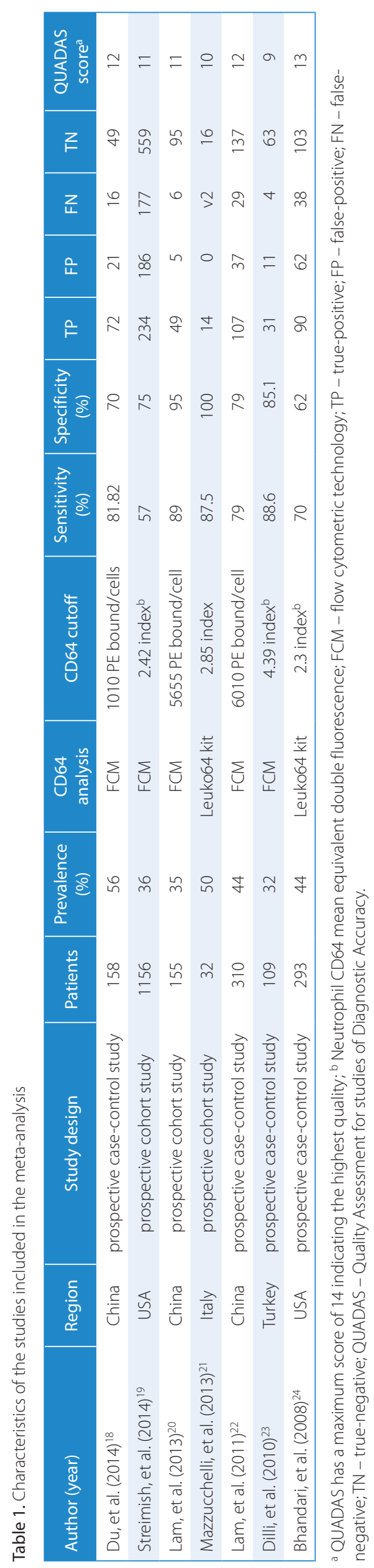




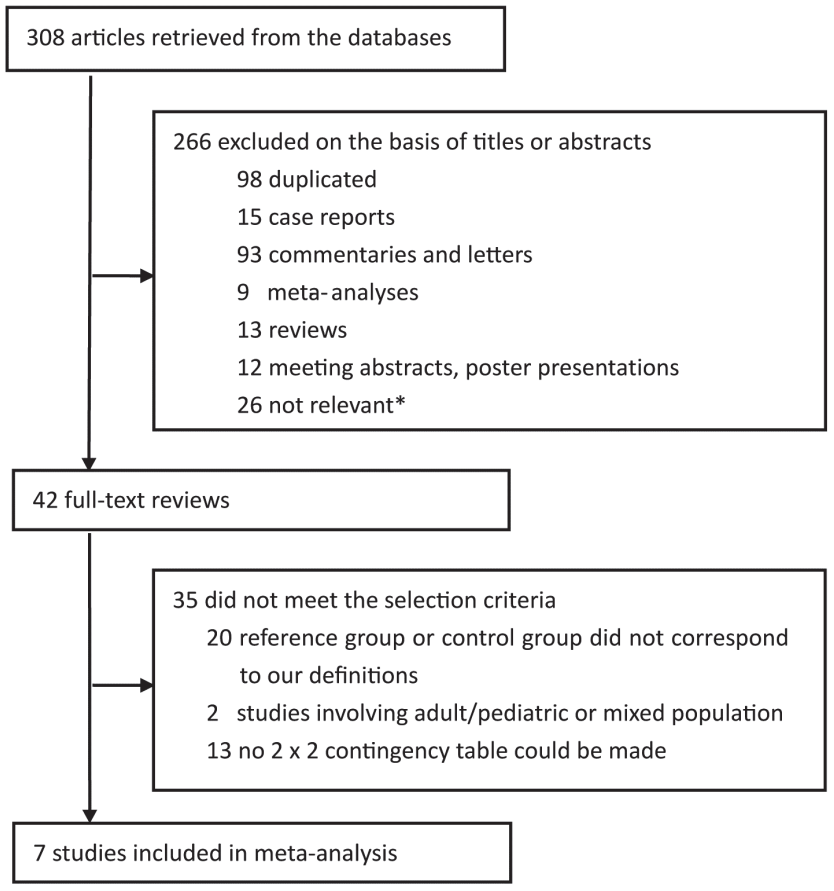

Fig. 1. Study selection. Some studies were excluded for more than one reason * Did not investigate the diagnostic accuracy of neutrophil CD64 as a marker for sepsis.

71-90\%) (Fig. 2). The pooled DOR was 19 (95\%CI, 6-57), whereas the pooled P/N LRs were 4.6 (95\%CI, 2.5-8.6) and 0.24 (95\%CI, 0.14-0.41), respectively. The area under the SROC curve for CD64 was 0.88 (95\%CI, 0.85-0.91) (Fig. 3). Fagan's nomogram for likelihood ratios indi- cated that using nCD64 expression to diagnose neonatal sepsis increased the post-probability to $54 \%$ when the results were positive and reduced the post-probability to $6 \%$ when the results were negative (Fig. 4). The effect of the diagnostic threshold was not significant (p-value $=0.71>0.05)$. Deek's funnel plot asymmetry test revealed the existence of publication bias with asymmetry in the data (p-value $=0.03<0.05)($ Fig. 5).

\section{Discussion}

Neonatal sepsis is one of the most common causes of neonatal deaths. Diagnosing neonatal sepsis is a serious challenge, because there is no single test that can be used for its early confirmation or exclusion. ${ }^{14,26}$ Recently, many researchers have focused on nCD64 as a marker of neonatal sepsis. ${ }^{8,16,17}$ In the light of this, the current meta-analysis was undertaken to estimate the efficiency of nCD64 for diagnosing neonatal sepsis.

As noted earlier, PCT is a very promising diagnostic marker of neonatal sepsis. ${ }^{27}$ The sensitivity of PCT is $81 \%$ and the specificity is $79 \%$. In the present study, the sensitivity and specificity of nCD64 were 80 and $83 \%$ respectively, which is similar to PCT. CRP is also an excellent marker and has been applied in clinical practice. ${ }^{28}$ The sensitivity of CRP ranges from 30 to $97 \%$, and the specificity ranges from 75 to $100 \% .{ }^{29}$ In the present meta-analysis, the sensitivity of nCD64 ranges from 57 to $89 \%$, and the specificity ranges from 62 to $100 \%$, indicating that $\mathrm{nCD} 64$ is a reli-

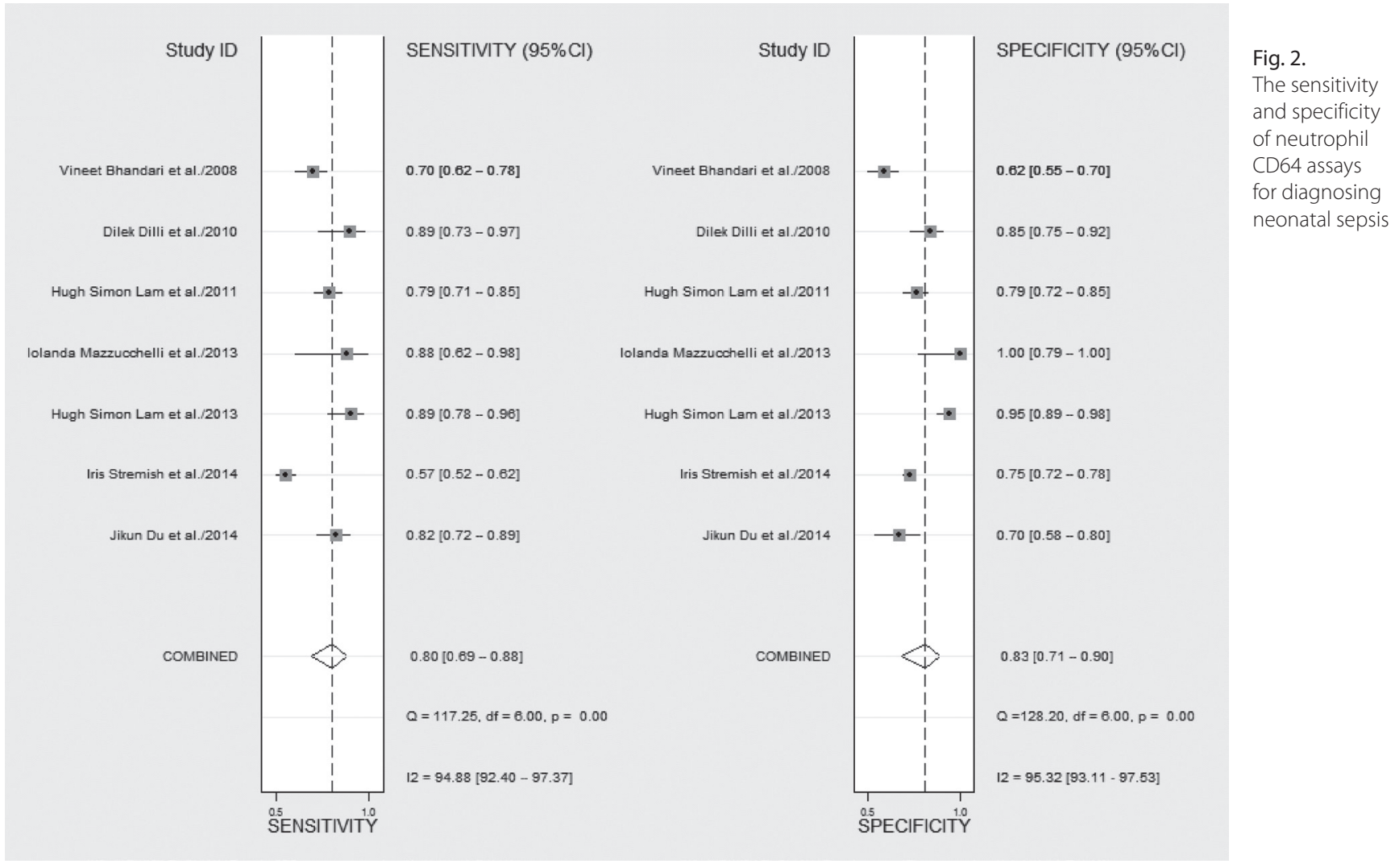




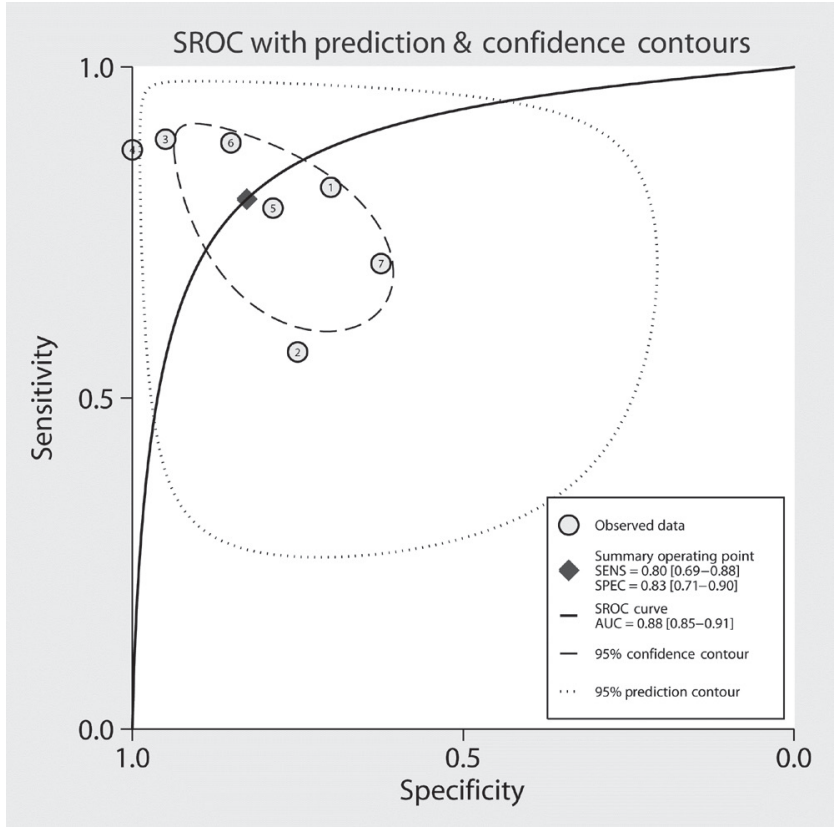

Fig. 3. The summary receiver-operating characteristic curve (SROC) showed a $95 \%$ confidence contour and $95 \%$ prediction contour

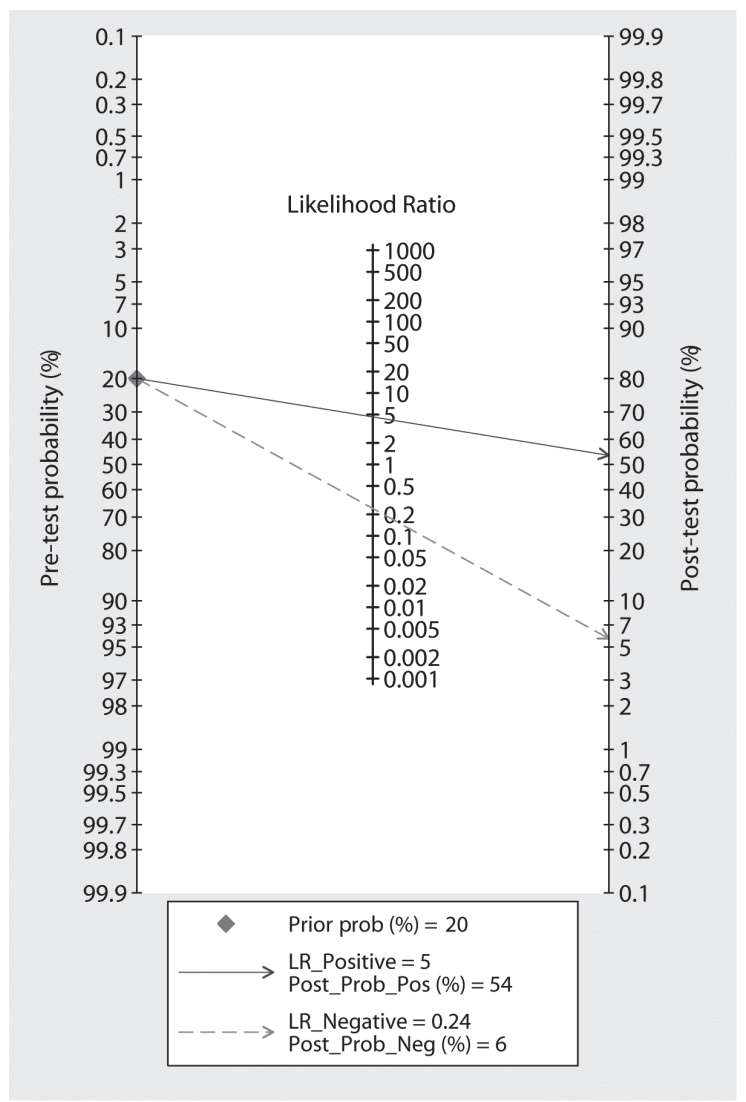

Fig. 4. Fagan's nomogram of the neutrophil CD64 test for diagnosing neonatal sepsis

able marker in the diagnosis of neonatal sepsis. Positive and negative likelihood ratios (P/N LRs) and post-test probability (PTP) are also relevant for clinicians. They both show whether a patient with a positive or negative

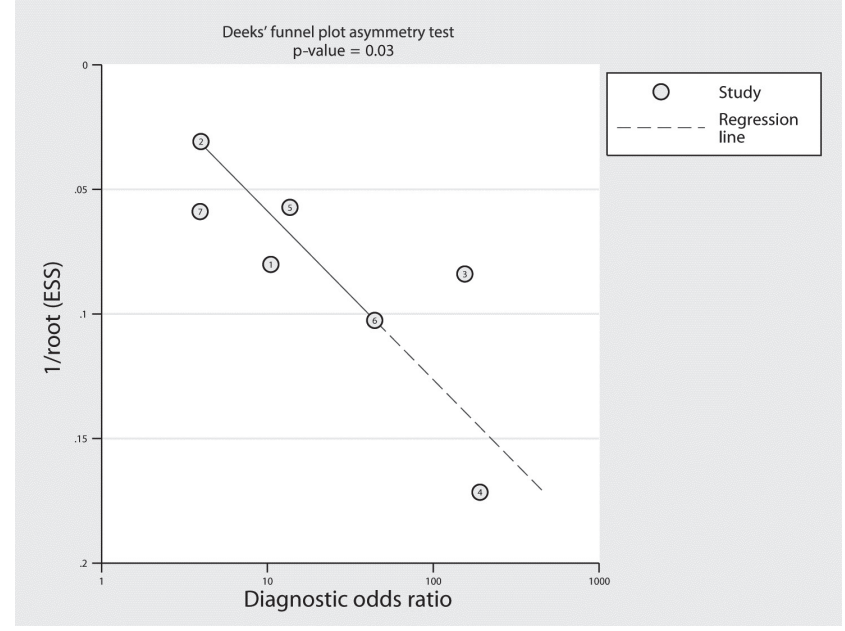

Fig. 5. Deek's funnel plot for the assessment of potential publication bias

test actually has sepsis or not. A PLR of 4.6 indicates that a neonatal with sepsis is 4.6 times more likely to have a positive test result than a neonatal without. The PTP for a positive test result is $54 \%$ with a given pretest probability of $20 \%$. Likewise, a NLR of 0.24 reduces the PTP to $6 \%$ for a negative result. The area under the SROC curve is 0.88 . However, significant statistical heterogeneity exists in the analysis $\left(\mathrm{I}^{2}=87.1 \%\right)$. Still, the interpretation of the above findings should not be ignored.

Several methods were tried to find the source of the high heterogeneity, including the threshold effect, publication bias, influence analysis and meta-regression. ${ }^{30}$ The different cutoff values for nCD64 did not account for the statistical heterogeneity through the analysis of threshold effect ( $p=0.71>0.05$ ). Deek's funnel plot asymmetry test showed the existence of publication bias (p-value $=0.03<0.05$ ), which is a source of heterogeneity. No valuable information was found through the sensitivity analysis and meta-regression. The meta-regression analysis included study design (prospective cohort or control-case study) and the gestational age of the neonates (preterm or not). The lack of a uniform definition of neonatal sepsis may potentially contribute to the high heterogeneity, especially for the clinically septic but culture-negative newborns, although the concept of clinical sepsis is widely used. This means that the selected studies use different criteria for the definition of sepsis. Thus, the spectrum of disorders and disease varies among the studies included. Considering that age may be a potential source of heterogeneity, studies in which over 15\% of the neonates were older than 28 days were excluded. Studies involving healthy neonates as controls in whom nCD64 will not be applied in routine clinical testing were also excluded, so they cannot be representative of the population studied in the current meta-analysis.

This meta-analysis has several limitations. First, substantial heterogeneity was detected among the studies included, but none of the study characteristics accounts 
for the majority of this heterogeneity. The studies differ in many ways, especially the definition criteria for neonatal sepsis and the postnatal age of the enrolled neonates. This important limitation will continue to exist in the research in this field until a uniform definition of neonatal sepsis is formulated. ${ }^{31,32}$ Second, a wide range of cut-off values in the reported $\mathrm{nCD} 64$ tests caused a wide variation in sensitivity and specificity. Even when the same method of measuring nCD64 expression was used, cut-off values were still different. ${ }^{21,23}$ Third, publication bias was detected. Studies with satisfactory results are more likely to be published, which can lead to overestimates of diagnostic accuracy. To overcome this problem, the authors searched again for further studies, but could not find additional relevant articles. Finally, only 1 study evaluated the performance of neutrophil CD64 in diagnosing EOS ${ }^{19}$ and only 2 evaluated it for LOS. ${ }^{21,22}$ The rest of the studies scarcely reported the percentage of EOS and LOS. So the accuracy of nCD64 for the diagnosis of EOS and LOS cannot be assessed.

In conclusion, nCD64 is a helpful marker for diagnosing neonatal sepsis. A study by Dhlamini et al. also shows that nCD64 has a high negative predictive value for excluding neonatal sepsis. ${ }^{33}$ But the results of nCD64 tests cannot be used alone to diagnose neonatal sepsis, as neonatal sepsis is a pathophysiological process rather than a specific syndrome and is too complex to be described by a single test. Further studies to determine the optimal cut-off values and to formulate a uniform definition of neonatal sepsis are urgently required.

\section{References}

1. Bizzarro MJ, Raskind C, Baltimore RS, et al. Seventy-five years of neonatal sepsis at Yale: 1928-2003. Pediatrics. 2005;116(3):595-602. doi: 10.1542/peds.2005-0552.

2. Stoll BJ, Hansen NI, Adams-Chapman I, et al. Neurodevelopmental and growth impairment among extremely low-birth-weight infants with neonatal infection. Jama. 2004;292(19):2357-2365. doi: 10.1001/jama.292.19.2357.

3. Lawn JE, Wilczynska-Ketende K, Cousens SN. Estimating the causes of 4 million neonatal deaths in the year 2000. Int J Epiemilol. 2006;35(3):706-718. doi: 10.1093/ije/dyl043.

4. Osrin D, Vergnano S, Costello A. Serious bacterial infections in newborn infants in developing countries. Curr Opin Infect Dis. 2004;17:217-224.

5. Persson E, Trollfors B, Brandberg LL, et al. Spticaemia and meningitis in neonates and during early infancy in the Göteborg area of Sweden. Acta Paediatr. 2002;91:1087-1092.

6. Sundaram V, Kumar P, Dutta S, et al. Blood culture confirmed bacterial sepsis in neonates in a North Indian tertiary care center: Changes over the last decade. Jpn J Infect Dis. 2009; 62(1):46-50.

7. Nupponen I, Andersson S, Järvenpää A-L, et al. Neutrophil CD11b expression and circulating interleukin-8 as diagnostic markers for early-onset neonatal sepsis. Pediatrics.2001; 108:e12-e12.

8. Layseca-Espinosa E, Pérez-González LF, Torres-Montes A, et al. Expression of CD64 as a potential marker of neonatal sepsis. Pediatr Allergy Immu.2002; 13:319-327.

9. Icardi M, Erickson Y, Kilborn S, et al. CD64 index provides simple and predictive testing for detection and monitoring of sepsis and bacterial infection in hospital patients. J Clinmicrobiol. 2009;47(12):3914-3919. doi: 10.1128/JCM.00628-09.

10. Ng P. Diagnostic markers of infection in neonates. Arch Dls Childfetal. 2004;89:F229-F235.
11. Kolackova M, Kudlova M, Kunes P, et al. Early expression of FcgammaRI (CD64) on monocytes of cardiac surgical patients and higher density of monocyte anti-inflammatory scavenger CD163 receptor in "on-pump" patients. Mediat Inflamm. 2007;2008(2008):235461. doi: $10.1155 / 2008 / 235461$.

12. Hoffmeyer F, Witte K, Schmidt R. The high-affinity FcyRI on PMN: Regulation of expression and signal transduction. Immunology. 1997;92:544-552.

13. van der Meer W, Pickkers $P$, Scott CS, et al. Hematological indices, inflammatory markers and neutrophil CD64 expression: Comparative trends during experimental human endotoxemia. J Endotoxin Res. 2007;13(2):94-100. doi: 10.1177/0968051907079101.

14. Ng PC, Lam HS. Diagnostic markers for neonatal sepsis. Curr Opin Pediatr. 2006;18:125-131.

15. Nuutila J, Hohenthal U, Laitinen I, et al. Simultaneous quantitative analysis of $\mathrm{Fc \gamma RI}(\mathrm{CD} 64)$ expression on neutrophils and monocytes: A new, improved way to detect infections. HImmunol Methods.2007;328:189-200.

16. Livaditi O, Kotanidou A, Psarra A, et al. Neutrophil CD64 expression and serum IL-8: Sensitive early markers of severity and outcome in sepsis. Cytokine. 2006;36:283-290.

17. Davis $\mathrm{BH}$, Olsen $\mathrm{SH}$, Ahmad E, et al. Neutrophil CD64 is an improved indicator of infection or sepsis in emergency department patients. ArCh Pathol Lab Med. 2006;130:654-661.

18. Whiting P, Rutjes AW, Reitsma JB,et al. The development of QUADAS: A tool for the quality assessment of studies of diagnostic accuracy included in systematic reviews. BMC Med Res Methodol.2003;3:25.

19. Du J, Li L, Dou Y, Li P, et al. Diagnostic utility of neutrophil CD64 as a marker for early-onset sepsis in preterm neonates. PloS one. 2014; 9(7):e102647. doi:10.1371/journal.pone.0102647.

20. Streimish I, Bizzarro M, Northrup V, et al. Neutrophil CD64 with hematologic criteria for diagnosis of neonatal sepsis. Am J Perinat. 2013;31:021-030.

21. Lam HS, Cheung HM, Poon TCW, et al. Neutrophil CD64 for daily surveillance of systemic infection and necrotizing enterocolitis in preterm infants. Clin Biochem. 2013;59:1753-1760.

22. Mazzucchelli I, Garofoli F, Ciardelli L, et al. Diagnostic performance of triggering receptor expressed on myeloid cells- 1 and CD64 index as markers of sepsis in preterm newborns. Pediatr Crit Care Me. 2013;14:178-182.

23. Lam HS, Wong SPS, Cheung HM, et al. Early diagnosis of intraabdominal inflammation and sepsis by neutrophil CD64 expression in newborns. Neonatology. 2010;99:118-124.

24. Dilli D, Oğuz şS, Dilmen Uu, Köker MY, et al. Predictive values of neutrophil CD64 expression compared with interleukin-6 and C-reactive protein in early diagnosis of neonatal sepsis. J Clin Lab Anal. 2010;24(6):363-370. doi: 10.1002/jcla.20370.

25. Bhandari V, Wang C, Rinder C, et al. Hematologic profile of sepsis in neonates: Neutrophil CD64 as a diagnostic marker. Pediatrics. 2008;121:129-134.

26. Magudumana $\mathrm{O}$, Ballot $\mathrm{D}$, Cooper $\mathrm{P}$, et al. Serial interleukin- 6 measurements in the early diagnosis of neonatal sepsis. $J$ Trop Pediatrics. 2000;46:267-271.

27. Vouloumanou EK, Plessa E, Karageorgopoulos DE, et al. Serum procalcitonin as a diagnostic marker for neonatal sepsis: A systematic review and meta-analysis. Intens Care Med. 2011;37(5):747-762. doi: 10.1007/s00134-011-2174-8.

28. Levy MM, Fink MP, Marshall JC, et al. 2001 SCCM/ESICM/ACCP/ATS/ SIS International Sepsis Definitions Conference. Intens Care Med. 2003:29:530-538.

29. Chan T, Gu F. Early diagnosis of sepsis using serum biomarkers. Expert Rev Mol Diagn. 2014;11:487-496.

30. Petitti DB. Approaches to heterogeneity in meta-analysis. Stat Med. 2001;20:3625-3633.

31. Committee ACP. Clinical policy for children younger than three years presenting to the emergency department with fever. Ann Emerg Med. 2003;42:530-545.

32. Goldstein B, Giroir B, Randolph A. International pediatric sepsis consensus conference: Definitions for sepsis and organ dysfunction in pediatrics. Pediatr Crit Care Me. 2005;6:2-8.

33. Dhlamini M, Suchard M, Wiggill T, et al. Neutrophil CD64 has a high negative predictive value for exclusion of neonatal sepsis. South African of Child Health. 2013;7:25-29. 